\title{
Radiological Assessment of Atmospheric Release from Tehran Research Reactor during Normal Operation by Using Pc-Cream Code
}

\author{
N. Sadeghi ${ }^{1, a^{*}}$, R. Ahangari ${ }^{2, b}$ \\ ${ }^{1,2}$ Reactor School-Nuclear Science and Technology Institute-Tehran-Iran \\ a nsadeghi@aeoi.org.ir, 'brahangarishahdehi@gmail.com
}

\begin{abstract}
Keywords: Normal Operation, Reactor Stack, Radioactive Material, Pc-Cream Code, Radiological Exposure Assessment, Radioactive Sample Analyzing
\end{abstract}

\begin{abstract}
In this work, radiological assessment of atmospheric release from Tehran's Research Reactor (TRR) stack and assessment of public exposures under normal operation has been studied. To perform tasks mentioned above, Pc-Cream computer code which simulates Gaussian Dispersion air transport plume model as well as laboratory analysis of the soil and leaves samples and TLD (Thermo Luminescent Dosimeter) monitoring around the TRR site was used. Results of the PcCream code showed that the annual committed and external dose received by the individual in the vicinity of the reactor is below the regulatory limit. Also, the results of laboratory analysis of available radionuclides in the soil and leaves samples showed that the concentrations are close to the background $\left(\mathrm{K} 40=635\right.$, Th232 $=28, \mathrm{Cs} 137=0.29$ up to $28.82, \mathrm{Ra} 226=25\left(\mathrm{~Bq}^{1} / \mathrm{Kg}\right)$ in soil and $\mathrm{K} 40=457, \mathrm{Be} 7 \approx 70(\mathrm{~Bq} / \mathrm{Kg})$ in leaves $)$ and confirm the code results. The monitored dose values of the TLD detectors were positioned around the reactor within $500 \mathrm{~m}$ radius shows that the background dose in vicinity of TRR (113 $\mu \mathrm{Sv}$ up to $150 \mu \mathrm{Sv})$ is consistent with the background dose in Tehran province (125 $\mu \mathrm{Sv})$.
\end{abstract}

\section{Introduction}

Tehran Research Reactor (TRR) is a 5 megawatt-thermal (MWth) pool-type research reactor in which light-water is used for cooling and moderation. TRR core configuration contains mixed material test reactor (MTR) plate-type fuel element. The TRR site is located at north of the Tehran city. The radioactive exposure in close proximity to the reactor site is one of the essential safety concerns in the nuclear reactors. Because of the unavoidable presence of personnel within the reactor site and the people outside of the site, radiological dose assessment should be performed as a matter of routine, albeit properly. Radiological dose assessments involve the calculation of radiation exposure which can achieve through the computer modeling and experimental measurements. The models describe the transformation of radionuclide in the environment. In this work, the researchers assess the radiological dose resulting from radioactive discharge into the environment by the Pc-Cream98 (Consequences of Release to the Environment Assessment

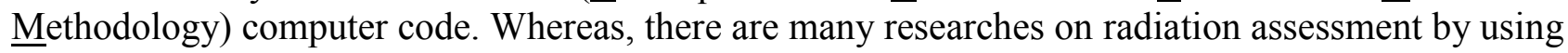
computational modeling Pc-Cream98 which done by other researches in different parts of the globe $[1,2,3,4]$, so it's a good method for assessing the radiological dose resulting from radioactive discharge into the environment of nuclear facilities.

Also, experimental measurements analysis of available radionuclide in the air, soil and leaves samples are performed to confirm the code calculations results.

\section{Materials and Methods}

In this study, situations of Tehran Research Reactor in the normal operation were assessed through the radionuclide discharge modeling and analysis of sample's data. For accuracy in dose prediction considering the people in the vicinity of TRR, in addition of the daily sampling from stack emission by the glass-fiber and charcoal filters and analyzing them, situations of TRR normal operation modeled by using of Pc-Cream98 computer code.

\footnotetext{
${ }^{1} \mathrm{~Bq}=$ Becquerel, is the $\mathrm{SI}$ derived unit of radioactivity.
} 


\section{Radionuclide dispersion modeling}

Radiological impact assessment can be achieved through the modeling in the absence of measurements. Calculations of dose projections for atmospheric releases during normal operation of TRR reactor were performed by using the assessor and plume part of Pc-Cream98 code. The methodology of Pc-Cream98 code is intended for use in assessing the radiological consequences to the persons in the vicinity of reactors, due to the discharge of radioactive effluents during normal operations. This code has been developed by the health protection agency (HPA), by the permission of the European Commission (EC) [5]. The Pc-Cream98 code includes revisions to the dosimetry and calculation of the effective dose, as described by ICRP 60 and using dose coefficients from ICRP 72 [6]. It uses a Gaussian model to describe the atmospheric dispersion of radioactive. The Gaussian plume model, which produces results that agree with experimental data as well as any model, is fairly easy to work with, and is consistent with the random nature of turbulence. It is also considered suitable where the end-points of the calculation are long-term average or time-integrated concentrations in air, as is normally the case in the assessment of dose from continuous releases. The Pc-Cream98 code required inputs data are provided by the Safety Analysis Report (SAR), Environmental report (ER) [7,8]. Annual data of wind speed, wind direction and wind stability class frequency are provided as inputs in Table 1.

Table 1 . Thermal stability classes and predominant wind direction

\begin{tabular}{|c|c|c|c|c|c|c|c|c|c|c|c|c|c|c|c|c|c|}
\hline & wind direction & $\begin{array}{l}n \\
\tilde{N}\end{array}$ & $\stackrel{n}{\vee}$ & $\frac{n}{\tilde{v}}$ & a & $\begin{array}{l}\stackrel{n}{\Xi} \\
\vec{v}\end{array}$ & $\stackrel{n}{\vec{v}}$ & $\frac{n}{\tilde{n}}$ & $\stackrel{\infty}{\nabla}$ & $\begin{array}{l}n \\
\stackrel{\mathbb{N}}{\mathbb{V}} \\
\end{array}$ & $\stackrel{\approx}{\hat{V}}$ & $\begin{array}{l}\stackrel{n}{\sim} \\
\stackrel{\sim}{\sim}\end{array}$ & $\underset{\widetilde{V}}{\stackrel{尺}{V}}$ & 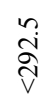 & $\frac{n}{v}$ & $\frac{n}{\tilde{n}}$ & $\begin{array}{l}\stackrel{8}{0} \\
\text { V }\end{array}$ \\
\hline & wind frequency & $\stackrel{t}{0}$ & $\stackrel{0}{0}$ & $\stackrel{8}{\circ}$ & $\stackrel{+}{0}$ & $\stackrel{m}{0}$ & $\stackrel{\infty}{\circ}$ & $\stackrel{\circ}{\circ}$ & $\stackrel{\circ}{\circ}$ & $\stackrel{\circ}{0}$ & $\stackrel{\circ}{\circ}$ & $\stackrel{n}{\circ}$ & $=$ & $\stackrel{\leftrightarrow}{\circ}$ & $\stackrel{3}{0}$ & 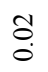 & $\stackrel{\text { ô }}{0}$ \\
\hline stability class & class frequency & & & & & & & & & & & & & & & & \\
\hline A & 0.1967 & 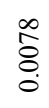 & $\begin{array}{l}\infty \\
\stackrel{8}{0} \\
0\end{array}$ & $\stackrel{\infty}{\stackrel{\infty}{\sigma}}$ & 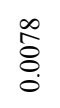 & 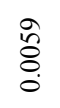 & $\frac{n}{a}$ & $\stackrel{\infty}{\stackrel{\infty}{0}}$ & $\begin{array}{l}\hat{a} \\
\stackrel{0}{0}\end{array}$ & $\stackrel{ }{\stackrel{5}{0}}$ & $\begin{array}{l}\stackrel{\infty}{m} \\
\stackrel{\overbrace{}}{0} \\
\stackrel{0}{0}\end{array}$ & 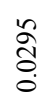 & $\begin{array}{l}\stackrel{0}{\pi} \\
\stackrel{\sigma}{\delta} \\
\dot{0}\end{array}$ & $\begin{array}{l}\infty \\
\stackrel{0}{0} \\
0\end{array}$ & ڤે & ڤે & ڤ్ڤ \\
\hline B & 0.0728 & సे. & $\begin{array}{l}0 \\
\tilde{\delta} \\
\delta \\
0\end{array}$ & $\begin{array}{l}\text { qे } \\
\stackrel{0}{0}\end{array}$ & 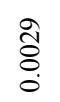 & $\begin{array}{l}\bar{\delta} \\
\stackrel{\delta}{\circ}\end{array}$ & 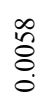 & 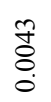 & 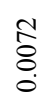 & $\begin{array}{l}n \\
\vdots \\
\vdots \\
0\end{array}$ & 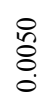 & $\stackrel{\circ}{\circ}$ & $\begin{array}{l}\circ \\
\stackrel{0}{0} \\
\stackrel{0}{0}\end{array}$ & 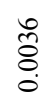 & $\begin{array}{l}\overrightarrow{\widehat{\delta}} \\
\stackrel{0}{0}\end{array}$ & 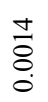 & $\stackrel{+}{\stackrel{\nabla}{\circ}}$ \\
\hline $\mathrm{C}$ & 0.0957 & $\begin{array}{l}\infty \\
\tilde{\delta} \\
0 \\
0\end{array}$ & $\begin{array}{l}\text { f } \\
8 \\
0\end{array}$ & $\begin{array}{l}\hat{n} \\
\vdots \\
0\end{array}$ & $\begin{array}{l}\infty \\
\stackrel{\infty}{8} \\
0\end{array}$ & $\begin{array}{l}\infty \\
\stackrel{\Xi}{8} \\
\stackrel{0}{0}\end{array}$ & $\begin{array}{l}0 \\
\stackrel{0}{8} \\
0 \\
0\end{array}$ & $\begin{array}{l}\tilde{a} \\
\vdots \\
0\end{array}$ & $\stackrel{\circ}{\circ}$ & $\begin{array}{l}0 \\
\stackrel{0}{0} \\
\stackrel{0}{0}\end{array}$ & 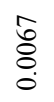 & $\begin{array}{l}\underset{f}{*} \\
\stackrel{\Xi}{\circ}\end{array}$ & $\begin{array}{l}\stackrel{n}{\sigma} \\
0 \\
0\end{array}$ & $\begin{array}{l}\text { f } \\
\stackrel{8}{0}\end{array}$ & $\begin{array}{l}\stackrel{\infty}{1} \\
\stackrel{8}{0} \\
\stackrel{0}{0}\end{array}$ & $\frac{9}{8}$ & $\stackrel{9}{8}$ \\
\hline D & 0.2416 & $\begin{array}{l}\text { \&̊ } \\
\text { ठ̊. }\end{array}$ & Iํ. & $\stackrel{\Delta}{\circ}$ & $\begin{array}{l}\text { \&े } \\
\stackrel{8}{0}\end{array}$ & $\begin{array}{l}\mathbb{N} \\
\stackrel{8}{8} \\
\stackrel{0}{0}\end{array}$ & $\frac{\mathfrak{2}}{\dot{\sigma}}$ & $\begin{array}{l}\stackrel{n}{+} \\
\stackrel{0}{0}\end{array}$ & $\underset{\stackrel{I}{J}}{\stackrel{I}{0}}$ & $\stackrel{\curvearrowright}{\widetilde{\Xi}}$ & $\begin{array}{l}\stackrel{0}{0} \\
0 \\
0\end{array}$ & 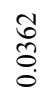 & 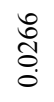 & $\underset{\vec{J}}{\overrightarrow{0}}$ & $\begin{array}{l}\mathbb{N} \\
\stackrel{8}{8} \\
\stackrel{0}{0}\end{array}$ & $\stackrel{\infty}{\stackrel{\infty}{8}}$ & \begin{tabular}{l}
$\infty$ \\
\multirow{8}{0}{} \\
$\stackrel{0}{0}$
\end{tabular} \\
\hline E & 0.1077 & $\begin{array}{l}\text { fo } \\
\stackrel{8}{0}\end{array}$ & $\begin{array}{l}\tilde{\delta} \\
\tilde{\delta}\end{array}$ & $\begin{array}{l}\text { ț } \\
\stackrel{0}{0} \\
0\end{array}$ & $\begin{array}{l}\stackrel{m}{8} \\
\stackrel{8}{0}\end{array}$ & $\begin{array}{l}\tilde{\delta} \\
\tilde{\delta} \\
0\end{array}$ & $\begin{array}{l}\stackrel{\circ}{\circ} \\
\stackrel{0}{0} \\
0\end{array}$ & $\begin{array}{l}\text { ț } \\
\stackrel{0}{0} \\
\stackrel{0}{0}\end{array}$ & $\stackrel{\infty}{\stackrel{\circ}{0}}$ & $\hat{o}$ & $\begin{array}{l}\hat{s} \\
\stackrel{8}{0} \\
\dot{0}\end{array}$ & $\begin{array}{l}\text { 임 } \\
\stackrel{0}{0}\end{array}$ & $\stackrel{\stackrel{9}{\overrightarrow{0}}}{\stackrel{0}{0}}$ & $\begin{array}{l}\tilde{\delta} \\
\stackrel{0}{0}\end{array}$ & $\begin{array}{l}\tilde{\delta} \\
\tilde{\delta} \\
0\end{array}$ & $\begin{array}{l}\overrightarrow{\widehat{\delta}} \\
\stackrel{0}{0}\end{array}$ & $\begin{array}{l}\vec{\delta} \\
\stackrel{\delta}{0}\end{array}$ \\
\hline $\mathrm{F}$ & 0.0890 & $\begin{array}{l}n \\
\tilde{\delta} \\
\dot{0}\end{array}$ & 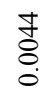 & $\begin{array}{l}\tilde{n} \\
\hat{\delta} \\
0 \\
\dot{0}\end{array}$ & $\begin{array}{l}\tilde{n} \\
\tilde{\delta} \\
\dot{0} \\
\dot{0}\end{array}$ & $\begin{array}{l}\text { İ } \\
\text { ¿े } \\
\stackrel{0}{0}\end{array}$ & $\begin{array}{l}\overrightarrow{5} \\
\stackrel{8}{0} \\
\dot{0}\end{array}$ & $\begin{array}{l}n \\
\hat{\delta} \\
0 \\
\dot{0}\end{array}$ & $\begin{array}{l}\stackrel{\otimes}{0} \\
\stackrel{0}{0}\end{array}$ & $\begin{array}{l}\stackrel{0}{ } \\
\stackrel{8}{0} \\
\dot{0}\end{array}$ & 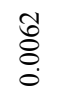 & 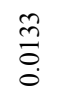 & $\begin{array}{l}\hat{8} \\
\dot{0} \\
\dot{0}\end{array}$ & $\begin{array}{l}\text { 莳 } \\
\stackrel{0}{0} \\
0\end{array}$ & 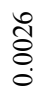 & $\begin{array}{l}\stackrel{0}{\circ} \\
\stackrel{8}{0}\end{array}$ & $\begin{array}{l}\stackrel{ }{\circ} \\
\stackrel{8}{0}\end{array}$ \\
\hline $\mathrm{C}$-rain & 0.1075 & $\stackrel{+}{8}$ & $\begin{array}{l}\tilde{\delta} \\
\hat{\delta} \\
0\end{array}$ & 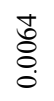 & $\stackrel{+}{8}$ & $\begin{array}{l}\tilde{O} \\
\stackrel{8}{0}\end{array}$ & $\begin{array}{l}\circ \\
\stackrel{8}{0} \\
\stackrel{0}{0}\end{array}$ & 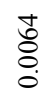 & $\stackrel{\circ}{\circ}$ & $\begin{array}{l}\text { \&े } \\
\dot{8}\end{array}$ & 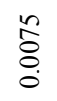 & $\begin{array}{l}\vec{\sigma} \\
0 \\
0\end{array}$ & $\stackrel{\infty}{\rightleftarrows}$ & $\begin{array}{l}\tilde{\delta} \\
\stackrel{0}{0}\end{array}$ & ஸै & $\begin{array}{l}\vec{\sigma} \\
\stackrel{0}{0}\end{array}$ & $\begin{array}{l}\bar{\delta} \\
\stackrel{0}{0}\end{array}$ \\
\hline B-rain & 0.0890 & 每 & 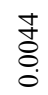 & $\begin{array}{l}\tilde{2} \\
\stackrel{0}{0} \\
0\end{array}$ & $\begin{array}{l}\tilde{n} \\
\tilde{\delta} \\
0\end{array}$ & 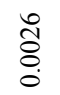 & 홍 & $\begin{array}{l}n \\
\stackrel{8}{0} \\
0\end{array}$ & $\begin{array}{l}\stackrel{0}{0} \\
\stackrel{0}{0}\end{array}$ & $\begin{array}{l}\stackrel{0}{ } \\
\stackrel{8}{0} \\
0\end{array}$ & $\begin{array}{l}\mathcal{O} \\
8 \\
0 \\
0\end{array}$ & $\begin{array}{l}\tilde{m} \\
\stackrel{0}{0}\end{array}$ & ôे & $\begin{array}{l}\text { 莳 } \\
\stackrel{0}{0}\end{array}$ & 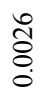 & $\frac{1}{8}$ & $\frac{1}{8}$ \\
\hline
\end{tabular}

The frequency of the wind speed and direction are reported by the Tehran nuclear research meteorological center. Distribution of wind speed and direction in 16 sectors around the TRR, demonstrated in Fig. 1. The dominant winds direction in TRR site is S\&W to N\&E and predominant wind speed is $4-6 \mathrm{~m} / \mathrm{s}$. 


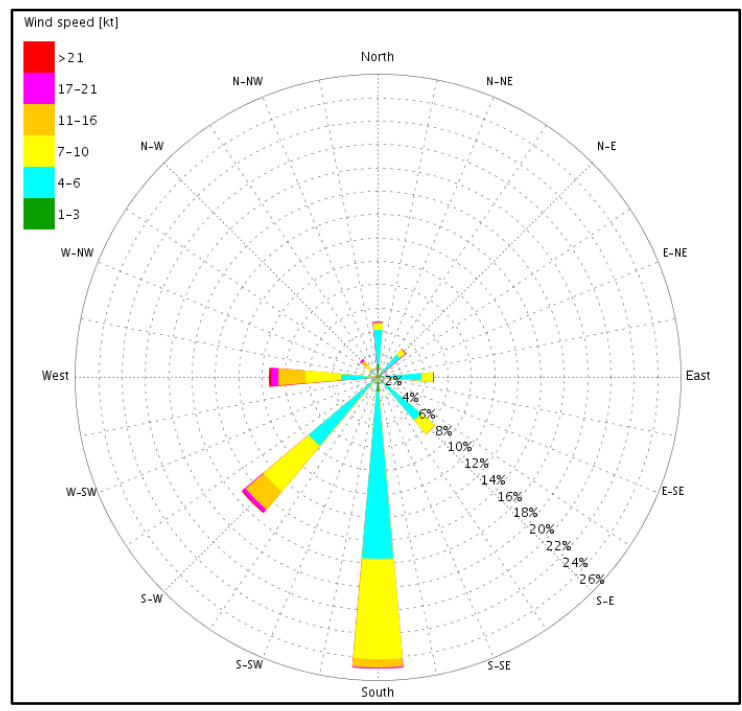

Fig. 1. Distribution of wind speed and direction in 16 sectors around the TRR

Also the site specific data, stack height, stack diameter and etc. which used as inputs demonstrate in Table 2.

Table 2. TRR site - specific data

\begin{tabular}{lc}
\hline Stack diameter / Stack height & $2.5 \mathrm{~m} / 57.0 \mathrm{~m}$ \\
\hline Average Air temp. in spring & $21^{\circ} \mathrm{c}$ \\
Average Air temp. in summer & $28^{\circ} \mathrm{c}$ \\
Average Air temp. in autumn & $11^{\circ} \mathrm{c}$ \\
Average Air temp. in winter & $5^{\circ} \mathrm{c}$ \\
\hline Mixing layer height & $100-1300 \mathrm{~m}$ \\
\hline outdoor Occupancy & 0.2 \\
\hline Met scheme & Pasquill \\
\hline
\end{tabular}

Release rate per year of radioactive materials from the reactor stack are provided in Table 3 according to SAR and ER for Tehran research reactor. Annual releases of these radionuclides were used as source term inputs in PC-CREAM 98 for the TRR.

Table 3. Activity of radionuclides released from TRR's stack during a year

\begin{tabular}{cc}
\hline Radioactive material & Release rate $(\mathrm{Bq} /$ year $)$ \\
\hline Kr-87 & $1.48 \mathrm{E}+12$ \\
\hline Kr-88 & $1.48 \mathrm{E}+12$ \\
\hline Co-60 & $7.77 \mathrm{E}+08$ \\
\hline Sr-90 & $2.22 \mathrm{E}+07$ \\
\hline Cs-134 & $2.22 \mathrm{E}+08$ \\
\hline Cs-137 & $5.55 \mathrm{E}+08$ \\
\hline Ce-144 & $8.88 \mathrm{E}+07$ \\
\hline Ar-41 & $3.37 \mathrm{E}+13$ \\
\hline $\mathrm{I}-133$ & $3.44 \mathrm{E}+07$ \\
\hline $\mathrm{I}-131$ & $8.51 \mathrm{E}+06$
\end{tabular}

$\mathrm{Kr}=$ krypton, $\mathrm{Co}=$ Cobalt, $\mathrm{Sr}=$ Strontium, $\mathrm{Cs}=$ Cesium, $\mathrm{Ce}=\mathrm{Cerium}, \mathrm{Ar}=$ Argon, $\mathrm{I}=$ Iodine

The dose assessment is calculated within $3500 \mathrm{~m}$ around the TRR reactor for five receptor distance $(300,500,1500,2500$ and $3500 \mathrm{~m})$ and for dominant wind direction. Also the outdoor occupancy of 0.2 (average of $4.8 \mathrm{~h}$ spent in TRR site every day for a year, by personnel and ordinary people) as proposed [9]. The default value of receptor height is $1.5 \mathrm{~m}$ and the breathing rate is 7300,5500 and $1900\left(\mathrm{~m}^{3} /\right.$ year $)$ for adult, child and infant respectively. The calculation of radiological doses was performed for the inhalation and external exposure pathways. 


\section{Radionuclide experimental measurements}

In this study, the TLD detectors are used to measuring the area dose in the vicinity of TRR exclusion area. TLD detectors are positioned in 6 stations around the TRR at 1 meter above the ground level and after every 2 months they are analyzed in laboratory.

Soil sampling from TRR site area have been done according to IAEA procedure [10]. Each season of year 5 soil sample from 5 different directions (mostly wind direction which is S\&W to $\mathrm{N} \& \mathrm{E}$ ) in $500 \mathrm{~m}$ radius from TRR sent to the laboratory and analyzed with gamma spectroscopy for any artificial or natural radioactivity.

The leaves of the trees in TRR site were sampled two times in a year according to IAEA procedure [10]. These samples were prepared in two ways, wet samples for iodine and dry samples for any probable radionuclide. These samples which were taken from different direction (mostly wind direction which is $\mathrm{S} \& \mathrm{~W}$ to $\mathrm{N} \& \mathrm{E}$ ) up to $500 \mathrm{~m}$ radius from TRR, were sent to the laboratory and analyzed with gamma spectroscopy for any artificial or natural radioactivity.

\section{Results and Discussion}

The results of atmospheric dispersion modeling of radionuclides in table 3 which are released during normal operation of TRR are shown in Table 4-6. The annual activity concentration results of each released radionuclide and their daughters in different distances from the TRR are presented in Table 4. Radionuclide concentration close to the discharge point is higher than farther distance points. The predicted concentration and integrated concentration in the environment are combined with pathway and dosimetric data to obtain the projected dose.

Table 4. Results of activity concentrations calculation in air $\left(\mathrm{Bq} / \mathrm{m}^{3}\right)$ with PC-CREAM 98 for all discharged nuclides

\begin{tabular}{|c|c|c|c|}
\hline & Nuclide & Parent & $\mathrm{Bq} / \mathrm{m}^{3}$ \\
\hline \multirow{12}{*}{ 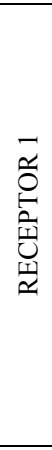 } & AR-41 & & $4.00 \mathrm{E}-01$ \\
\hline & $\mathrm{CO}-60$ & & $9.30 \mathrm{E}-06$ \\
\hline & KR-87 & & $1.70 \mathrm{E}-02$ \\
\hline & KR-88 & & $1.80 \mathrm{E}-02$ \\
\hline & SR-90 & & $2.70 \mathrm{E}-07$ \\
\hline & I-131 & & $1.00 \mathrm{E}-07$ \\
\hline & $\mathrm{I}-133$ & & $4.10 \mathrm{E}-07$ \\
\hline & CS-134 & & $2.70 \mathrm{E}-06$ \\
\hline & CS-137 & & $6.70 \mathrm{E}-06$ \\
\hline & CE-144 & & $1.10 \mathrm{E}-06$ \\
\hline & Y-90D & SR-90 & $1.40 \mathrm{E}-10$ \\
\hline & PR-144D & CE-144 & $1.10 \mathrm{E}-07$ \\
\hline \multirow{12}{*}{ 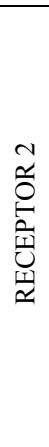 } & AR-41 & & $2.70 \mathrm{E}-01$ \\
\hline & $\mathrm{CO}-60$ & & $6.40 \mathrm{E}-06$ \\
\hline & KR-87 & & $1.20 \mathrm{E}-02$ \\
\hline & KR-88 & & $1.20 \mathrm{E}-02$ \\
\hline & SR-90 & & $1.80 \mathrm{E}-07$ \\
\hline & $\mathrm{I}-131$ & & $6.90 \mathrm{E}-08$ \\
\hline & I-133 & & $2.80 \mathrm{E}-07$ \\
\hline & CS-134 & & $1.80 \mathrm{E}-06$ \\
\hline & CS-137 & & $4.60 \mathrm{E}-06$ \\
\hline & CE-144 & & $7.30 \mathrm{E}-07$ \\
\hline & Y-90D & SR-90 & $1.40 \mathrm{E}-10$ \\
\hline & PR-144D & CE-144 & $1.10 \mathrm{E}-07$ \\
\hline \multirow{7}{*}{ 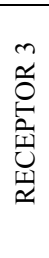 } & AR-41 & & $7.40 \mathrm{E}-02$ \\
\hline & $\mathrm{CO}-60$ & & $1.80 \mathrm{E}-06$ \\
\hline & KR-87 & & $3.20 \mathrm{E}-03$ \\
\hline & KR-88 & & $3.30 \mathrm{E}-03$ \\
\hline & SR-90 & & $5.10 \mathrm{E}-08$ \\
\hline & $\mathrm{I}-131$ & & $1.90 \mathrm{E}-08$ \\
\hline & I-133 & & $7.80 \mathrm{E}-08$ \\
\hline
\end{tabular}




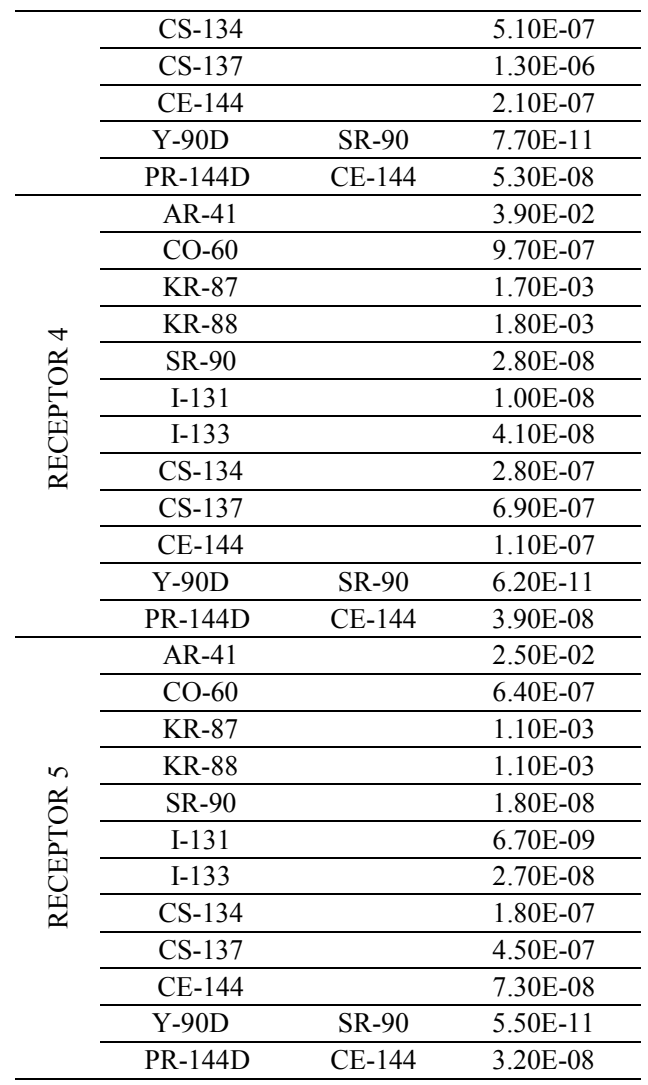

The results of committed individual doses from inhalation, gamma cloud shine, gamma deposition, re-suspension, beta cloud shine, beta deposition doses and external doses of these radionuclides in 1 year for adult are inserted in Table 5 and 6 respectively. The maximum value of the total committed dose for adults is $0.28 \mu \mathrm{Sv} / \mathrm{y}$ in the receptor 1 location $(300 \mathrm{~m})$. Also, the maximum amount of total external dose is $1.04 \mu \mathrm{Sv} / \mathrm{y}$ for this location. As the results show, the individual dose decreases with increasing the distance from the reactor stack.

Table 5. Results of committed individual doses calculation in year 1 with PC-CREAM 98 for adult in 5 distances around the TRR, $(\mu \mathrm{Sv})$

\begin{tabular}{|c|c|c|c|c|c|c|c|c|c|}
\hline \multirow{14}{*}{ 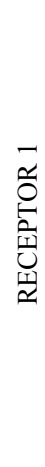 } & Nuclide & Parent & Inhalation & Cld. Gamma & Dep. Gamma & Resus. & Cld. Beta & Dep. Beta & Total \\
\hline & AR-41 & & $0.00 \mathrm{E}+00$ & $2.50 \mathrm{E}-01$ & $0.00 \mathrm{E}+00$ & $0.00 \mathrm{E}+00$ & $3.00 \mathrm{E}-03$ & $0.00 \mathrm{E}+00$ & $2.53 \mathrm{E}-01$ \\
\hline & CO-60 & & $6.80 \mathrm{E}-04$ & 1.10E-05 & $4.00 \mathrm{E}-03$ & $1.30 \mathrm{E}-06$ & $1.30 \mathrm{E}-08$ & $0.00 \mathrm{E}+00$ & $4.69 \mathrm{E}-03$ \\
\hline & KR-87 & & $0.00 \mathrm{E}+00$ & $6.50 \mathrm{E}-03$ & $0.00 \mathrm{E}+00$ & $0.00 \mathrm{E}+00$ & $3.60 \mathrm{E}-04$ & $0.00 \mathrm{E}+00$ & $6.86 \mathrm{E}-03$ \\
\hline & KR-88 & & $0.00 \mathrm{E}+00$ & $1.60 \mathrm{E}-02$ & $0.00 \mathrm{E}+00$ & $0.00 \mathrm{E}+00$ & $1.00 \mathrm{E}-04$ & $0.00 \mathrm{E}+00$ & $1.61 \mathrm{E}-02$ \\
\hline & SR-90 & & $7.00 \mathrm{E}-05$ & $0.00 \mathrm{E}+00$ & $2.60 \mathrm{E}-12$ & $1.30 \mathrm{E}-07$ & $8.00 \mathrm{E}-10$ & $4.10 \mathrm{E}-07$ & $7.05 \mathrm{E}-05$ \\
\hline & I-131 & & $5.50 \mathrm{E}-06$ & $2.10 \mathrm{E}-08$ & $1.80 \mathrm{E}-06$ & $1.40 \mathrm{E}-08$ & $3.50 \mathrm{E}-10$ & $5.50 \mathrm{E}-07$ & $7.89 \mathrm{E}-06$ \\
\hline & I-133 & & $4.50 \mathrm{E}-06$ & $1.30 \mathrm{E}-07$ & $1.30 \mathrm{E}-06$ & $1.80 \mathrm{E}-09$ & $3.00 \mathrm{E}-09$ & $1.50 \mathrm{E}-05$ & $2.09 \mathrm{E}-05$ \\
\hline & CS-134 & & $1.30 \mathrm{E}-04$ & $2.10 \mathrm{E}-06$ & $6.90 \mathrm{E}-04$ & $2.30 \mathrm{E}-07$ & $7.70 \mathrm{E}-09$ & $4.50 \mathrm{E}-06$ & $8.27 \mathrm{E}-04$ \\
\hline & CS-137 & & $2.20 \mathrm{E}-04$ & $1.90 \mathrm{E}-06$ & $7.30 \mathrm{E}-04$ & $4.30 \mathrm{E}-07$ & $2.80 \mathrm{E}-08$ & $2.00 \mathrm{E}-05$ & $9.72 \mathrm{E}-04$ \\
\hline & CE-144 & & $2.80 \mathrm{E}-04$ & $1.20 \mathrm{E}-08$ & $7.00 \mathrm{E}-06$ & $4.70 \mathrm{E}-07$ & $1.30 \mathrm{E}-09$ & $0.00 \mathrm{E}+00$ & $2.87 \mathrm{E}-04$ \\
\hline & Y-90D & SR-90 & $1.50 \mathrm{E}-09$ & $2.10 \mathrm{E}-18$ & $0.00 \mathrm{E}+00$ & $0.00 \mathrm{E}+00$ & $2.10 \mathrm{E}-12$ & $1.80 \mathrm{E}-09$ & $3.30 \mathrm{E}-09$ \\
\hline & PR-144D & CE-144 & $1.50 \mathrm{E}-08$ & 1.30E-09 & $0.00 \mathrm{E}+00$ & $0.00 \mathrm{E}+00$ & $2.20 \mathrm{E}-09$ & $1.90 \mathrm{E}-06$ & $1.92 \mathrm{E}-06$ \\
\hline & Total & & $1.39 \mathrm{E}-03$ & $2.73 \mathrm{E}-01$ & $5.43 \mathrm{E}-03$ & $2.58 \mathrm{E}-06$ & $3.46 \mathrm{E}-03$ & $4.24 \mathrm{E}-05$ & $2.83 \mathrm{E}-01$ \\
\hline \multirow{13}{*}{ 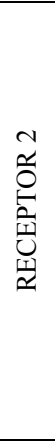 } & AR-41 & & $0.00 \mathrm{E}+00$ & $1.50 \mathrm{E}-01$ & $0.00 \mathrm{E}+00$ & $0.00 \mathrm{E}+00$ & $2.10 \mathrm{E}-03$ & $0.00 \mathrm{E}+00$ & $1.52 \mathrm{E}-01$ \\
\hline & CO-60 & & $4.70 \mathrm{E}-04$ & $6.70 \mathrm{E}-06$ & $2.50 \mathrm{E}-03$ & $8.00 \mathrm{E}-07$ & $8.70 \mathrm{E}-09$ & $0.00 \mathrm{E}+00$ & $2.98 \mathrm{E}-03$ \\
\hline & KR-87 & & $0.00 \mathrm{E}+00$ & $3.80 \mathrm{E}-03$ & $0.00 \mathrm{E}+00$ & $0.00 \mathrm{E}+00$ & $2.50 \mathrm{E}-04$ & $0.00 \mathrm{E}+00$ & $4.05 \mathrm{E}-03$ \\
\hline & KR-88 & & $0.00 \mathrm{E}+00$ & $9.40 \mathrm{E}-03$ & $0.00 \mathrm{E}+00$ & $0.00 \mathrm{E}+00$ & $7.00 \mathrm{E}-05$ & $0.00 \mathrm{E}+00$ & $9.47 \mathrm{E}-03$ \\
\hline & SR-90 & & $4.80 \mathrm{E}-05$ & $0.00 \mathrm{E}+00$ & $1.60 \mathrm{E}-12$ & $8.40 \mathrm{E}-08$ & $5.50 \mathrm{E}-10$ & $2.60 \mathrm{E}-07$ & $4.83 \mathrm{E}-05$ \\
\hline & I-131 & & $3.70 \mathrm{E}-06$ & $1.20 \mathrm{E}-08$ & $1.20 \mathrm{E}-06$ & $9.00 \mathrm{E}-09$ & $2.40 \mathrm{E}-10$ & $3.70 \mathrm{E}-07$ & $5.29 \mathrm{E}-06$ \\
\hline & I-133 & & $3.10 \mathrm{E}-06$ & $7.40 \mathrm{E}-08$ & $8.90 \mathrm{E}-07$ & $1.20 \mathrm{E}-09$ & $2.00 \mathrm{E}-09$ & $1.00 \mathrm{E}-05$ & $1.41 \mathrm{E}-05$ \\
\hline & $\begin{array}{l}\text { CS-134 } \\
\end{array}$ & & $8.80 \mathrm{E}-05$ & $1.20 \mathrm{E}-06$ & $4.30 \mathrm{E}-04$ & $1.50 \mathrm{E}-07$ & $5.20 \mathrm{E}-09$ & $2.80 \mathrm{E}-06$ & $5.22 \mathrm{E}-04$ \\
\hline & CS-137 & & $1.50 \mathrm{E}-04$ & $1.10 \mathrm{E}-06$ & $4.60 \mathrm{E}-04$ & $2.70 \mathrm{E}-07$ & $1.90 \mathrm{E}-08$ & $1.20 \mathrm{E}-05$ & $6.23 \mathrm{E}-04$ \\
\hline & CE-144 & & $1.90 \mathrm{E}-04$ & $6.50 \mathrm{E}-09$ & $4.40 \mathrm{E}-06$ & $3.00 \mathrm{E}-07$ & $8.70 \mathrm{E}-10$ & $0.00 \mathrm{E}+00$ & $1.95 \mathrm{E}-04$ \\
\hline & Y-90D & SR-90 & $1.50 \mathrm{E}-09$ & $2.10 \mathrm{E}-18$ & $0.00 \mathrm{E}+00$ & $0.00 \mathrm{E}+00$ & $2.00 \mathrm{E}-12$ & $1.70 \mathrm{E}-09$ & $3.20 \mathrm{E}-09$ \\
\hline & PR-144D & CE-144 & $1.40 \mathrm{E}-08$ & $1.20 \mathrm{E}-09$ & $0.00 \mathrm{E}+00$ & $0.00 \mathrm{E}+00$ & $2.10 \mathrm{E}-09$ & $1.80 \mathrm{E}-06$ & $1.82 \mathrm{E}-06$ \\
\hline & Total & & 9.53E-04 & $1.63 \mathrm{E}-01$ & $3.40 \mathrm{E}-03$ & $1.61 \mathrm{E}-06$ & $2.42 \mathrm{E}-03$ & 2.72E-05 & $1.70 \mathrm{E}-01$ \\
\hline
\end{tabular}




\begin{tabular}{|c|c|c|c|c|c|c|c|c|c|}
\hline \multirow{13}{*}{ 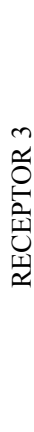 } & AR-41 & & $0.00 \mathrm{E}+00$ & $3.60 \mathrm{E}-02$ & $0.00 \mathrm{E}+00$ & $0.00 \mathrm{E}+00$ & $5.70 \mathrm{E}-04$ & $0.00 \mathrm{E}+00$ & $3.66 \mathrm{E}-02$ \\
\hline & $\mathrm{CO}-60$ & & $1.30 \mathrm{E}-04$ & $1.70 \mathrm{E}-06$ & $7.80 \mathrm{E}-04$ & $2.50 \mathrm{E}-07$ & $2.40 \mathrm{E}-09$ & $0.00 \mathrm{E}+00$ & $9.12 \mathrm{E}-04$ \\
\hline & KR-87 & & $0.00 \mathrm{E}+00$ & $9.50 \mathrm{E}-04$ & $0.00 \mathrm{E}+00$ & $0.00 \mathrm{E}+00$ & $6.70 \mathrm{E}-05$ & $0.00 \mathrm{E}+00$ & $1.02 \mathrm{E}-03$ \\
\hline & KR-88 & & $0.00 \mathrm{E}+00$ & $2.40 \mathrm{E}-03$ & $0.00 \mathrm{E}+00$ & $0.00 \mathrm{E}+00$ & $1.90 \mathrm{E}-05$ & $0.00 \mathrm{E}+00$ & $2.42 \mathrm{E}-03$ \\
\hline & SR-90 & & $1.40 \mathrm{E}-05$ & $0.00 \mathrm{E}+00$ & $5.10 \mathrm{E}-13$ & $2.60 \mathrm{E}-08$ & $1.50 \mathrm{E}-10$ & $8.10 \mathrm{E}-08$ & $1.41 \mathrm{E}-05$ \\
\hline & I-131 & & $1.00 \mathrm{E}-06$ & $2.90 \mathrm{E}-09$ & $3.40 \mathrm{E}-07$ & $2.60 \mathrm{E}-09$ & $6.60 \mathrm{E}-11$ & $1.10 \mathrm{E}-07$ & $1.46 \mathrm{E}-06$ \\
\hline & I-133 & & $8.50 \mathrm{E}-07$ & $1.80 \mathrm{E}-08$ & $2.50 \mathrm{E}-07$ & $3.40 \mathrm{E}-10$ & $5.60 \mathrm{E}-10$ & $2.90 \mathrm{E}-06$ & $4.02 \mathrm{E}-06$ \\
\hline & CS-134 & & $2.50 \mathrm{E}-05$ & $3.10 \mathrm{E}-07$ & $1.30 \mathrm{E}-04$ & $4.60 \mathrm{E}-08$ & $1.50 \mathrm{E}-09$ & $8.80 \mathrm{E}-07$ & $1.56 \mathrm{E}-04$ \\
\hline & CS-137 & & $4.30 \mathrm{E}-05$ & $2.80 \mathrm{E}-07$ & $1.40 \mathrm{E}-04$ & $8.40 \mathrm{E}-08$ & $5.30 \mathrm{E}-09$ & $3.80 \mathrm{E}-06$ & $1.87 \mathrm{E}-04$ \\
\hline & CE-144 & & $5.40 \mathrm{E}-05$ & $1.60 \mathrm{E}-09$ & $1.40 \mathrm{E}-06$ & $9.20 \mathrm{E}-08$ & $2.40 \mathrm{E}-10$ & $0.00 \mathrm{E}+00$ & $5.55 \mathrm{E}-05$ \\
\hline & Y-90D & SR-90 & $8.40 \mathrm{E}-10$ & $1.20 \mathrm{E}-18$ & $0.00 \mathrm{E}+00$ & $0.00 \mathrm{E}+00$ & $1.10 \mathrm{E}-12$ & $1.20 \mathrm{E}-09$ & $2.04 \mathrm{E}-09$ \\
\hline & PR-144D & CE-144 & $7.00 \mathrm{E}-09$ & $6.70 \mathrm{E}-10$ & $0.00 \mathrm{E}+00$ & $0.00 \mathrm{E}+00$ & $1.00 \mathrm{E}-09$ & $1.20 \mathrm{E}-06$ & $1.21 \mathrm{E}-06$ \\
\hline & Total & & $2.68 \mathrm{E}-04$ & $3.94 \mathrm{E}-02$ & $1.05 \mathrm{E}-03$ & \begin{tabular}{|l|l|}
$5.01 E-07$ \\
\end{tabular} & $\begin{array}{l}6.56 \mathrm{E}-04 \\
\end{array}$ & $8.97 \mathrm{E}-06$ & $4.13 \mathrm{E}-02$ \\
\hline \multirow{13}{*}{ 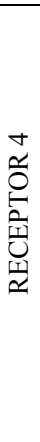 } & AR-41 & & $0.00 \mathrm{E}+00$ & $1.80 \mathrm{E}-02$ & $0.00 \mathrm{E}+00$ & $0.00 \mathrm{E}+00$ & $3.00 \mathrm{E}-04$ & $0.00 \mathrm{E}+00$ & $1.83 \mathrm{E}-02$ \\
\hline & CO-60 & & $7.10 \mathrm{E}-05$ & $8.80 \mathrm{E}-07$ & $4.50 \mathrm{E}-04$ & $1.40 \mathrm{E}-07$ & $1.30 \mathrm{E}-09$ & $0.00 \mathrm{E}+00$ & $5.22 \mathrm{E}-04$ \\
\hline & KR-87 & & $0.00 \mathrm{E}+00$ & $4.70 \mathrm{E}-04$ & $0.00 \mathrm{E}+00$ & $0.00 \mathrm{E}+00$ & $3.50 \mathrm{E}-05$ & $0.00 \mathrm{E}+00$ & $5.05 \mathrm{E}-04$ \\
\hline & KR-88 & & $0.00 \mathrm{E}+00$ & $1.20 \mathrm{E}-03$ & $0.00 \mathrm{E}+00$ & $0.00 \mathrm{E}+00$ & $1.00 \mathrm{E}-05$ & $0.00 \mathrm{E}+00$ & $1.21 \mathrm{E}-03$ \\
\hline & SR-90 & & $7.30 \mathrm{E}-06$ & $0.00 \mathrm{E}+00$ & $2.90 \mathrm{E}-13$ & $1.50 \mathrm{E}-08$ & $8.40 \mathrm{E}-11$ & $4.70 \mathrm{E}-08$ & $7.36 \mathrm{E}-06$ \\
\hline & I-131 & & $5.60 \mathrm{E}-07$ & $1.50 \mathrm{E}-09$ & $1.80 \mathrm{E}-07$ & $1.40 \mathrm{E}-09$ & $3.50 \mathrm{E}-11$ & $5.70 \mathrm{E}-08$ & $8.00 \mathrm{E}-07$ \\
\hline & I-133 & & $4.50 \mathrm{E}-07$ & $9.30 \mathrm{E}-09$ & $1.40 \mathrm{E}-07$ & $1.80 \mathrm{E}-10$ & $3.00 \mathrm{E}-10$ & $1.60 \mathrm{E}-06$ & $2.20 \mathrm{E}-06$ \\
\hline & CS-134 & & $1.30 \mathrm{E}-05$ & $1.60 \mathrm{E}-07$ & 7.70E-05 & $2.60 \mathrm{E}-08$ & $8.00 \mathrm{E}-10$ & $5.00 \mathrm{E}-07$ & $9.07 \mathrm{E}-05$ \\
\hline & CS-137 & & $2.30 \mathrm{E}-05$ & $1.40 \mathrm{E}-07$ & $8.20 \mathrm{E}-05$ & $4.80 \mathrm{E}-08$ & $2.90 \mathrm{E}-09$ & $2.20 \mathrm{E}-06$ & $1.07 \mathrm{E}-04$ \\
\hline & CE-144 & & $2.90 \mathrm{E}-05$ & $8.00 \mathrm{E}-10$ & $7.90 \mathrm{E}-07$ & $5.30 \mathrm{E}-08$ & $1.30 \mathrm{E}-10$ & $0.00 \mathrm{E}+00$ & $2.98 \mathrm{E}-05$ \\
\hline & Y-90D & SR-90 & $6.80 \mathrm{E}-10$ & $9.60 \mathrm{E}-19$ & $0.00 \mathrm{E}+00$ & $0.00 \mathrm{E}+00$ & $9.20 \mathrm{E}-13$ & $1.10 \mathrm{E}-09$ & $1.78 \mathrm{E}-09$ \\
\hline & PR-144D & CE-144 & $5.10 \mathrm{E}-09$ & $4.80 \mathrm{E}-10$ & $0.00 \mathrm{E}+00$ & $0.00 \mathrm{E}+00$ & $7.50 \mathrm{E}-10$ & $9.90 \mathrm{E}-07$ & $9.96 \mathrm{E}-07$ \\
\hline & Total & & $1.44 \mathrm{E}-04$ & $1.97 \mathrm{E}-02$ & $6.10 \mathrm{E}-04$ & 2.84E-07 & $3.45 \mathrm{E}-04$ & 5.40E-06 & $2.08 \mathrm{E}-02$ \\
\hline \multirow{13}{*}{ 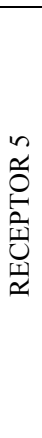 } & AR-41 & & $0.00 \mathrm{E}+00$ & $1.10 \mathrm{E}-02$ & $0.00 \mathrm{E}+00$ & $0.00 \mathrm{E}+00$ & $1.90 \mathrm{E}-04$ & $0.00 \mathrm{E}+00$ & $1.12 \mathrm{E}-02$ \\
\hline & CO-60 & & $4.60 \mathrm{E}-05$ & $5.60 \mathrm{E}-07$ & $3.10 \mathrm{E}-04$ & $1.00 \mathrm{E}-07$ & $8.70 \mathrm{E}-10$ & $0.00 \mathrm{E}+00$ & $3.57 \mathrm{E}-04$ \\
\hline & KR-87 & & $0.00 \mathrm{E}+00$ & $2.90 \mathrm{E}-04$ & $0.00 \mathrm{E}+00$ & $0.00 \mathrm{E}+00$ & $2.20 \mathrm{E}-05$ & $0.00 \mathrm{E}+00$ & $3.12 \mathrm{E}-04$ \\
\hline & KR-88 & & $0.00 \mathrm{E}+00$ & $7.60 \mathrm{E}-04$ & $0.00 \mathrm{E}+00$ & $0.00 \mathrm{E}+00$ & $6.70 \mathrm{E}-06$ & $0.00 \mathrm{E}+00$ & 7.67E-04 \\
\hline & SR-90 & & $4.80 \mathrm{E}-06$ & $0.00 \mathrm{E}+00$ & $2.00 \mathrm{E}-13$ & $1.00 \mathrm{E}-08$ & $5.50 \mathrm{E}-11$ & $3.20 \mathrm{E}-08$ & $4.84 \mathrm{E}-06$ \\
\hline & I-131 & & $3.60 \mathrm{E}-07$ & $9.20 \mathrm{E}-10$ & $1.20 \mathrm{E}-07$ & $9.30 \mathrm{E}-10$ & $2.30 \mathrm{E}-11$ & $3.80 \mathrm{E}-08$ & $5.20 \mathrm{E}-07$ \\
\hline & I-133 & & $2.90 \mathrm{E}-07$ & $5.90 \mathrm{E}-09$ & $9.10 \mathrm{E}-08$ & $1.20 \mathrm{E}-10$ & $1.90 \mathrm{E}-10$ & $1.00 \mathrm{E}-06$ & $1.39 \mathrm{E}-06$ \\
\hline & CS-134 & & $8.70 \mathrm{E}-06$ & $1.00 \mathrm{E}-07$ & $5.30 \mathrm{E}-05$ & $1.80 \mathrm{E}-08$ & $5.20 \mathrm{E}-10$ & $3.50 \mathrm{E}-07$ & $6.22 \mathrm{E}-05$ \\
\hline & CS-137 & & $1.50 \mathrm{E}-05$ & $9.20 \mathrm{E}-08$ & $5.70 \mathrm{E}-05$ & $3.30 \mathrm{E}-08$ & $1.90 \mathrm{E}-09$ & $1.50 \mathrm{E}-06$ & $7.36 \mathrm{E}-05$ \\
\hline & CE-144 & & $1.90 \mathrm{E}-05$ & $5.10 \mathrm{E}-10$ & $5.50 \mathrm{E}-07$ & $3.70 \mathrm{E}-08$ & $8.70 \mathrm{E}-11$ & $0.00 \mathrm{E}+00$ & $1.96 \mathrm{E}-05$ \\
\hline & Y-90D & SR-90 & $6.00 \mathrm{E}-10$ & $8.50 \mathrm{E}-19$ & $0.00 \mathrm{E}+00$ & $0.00 \mathrm{E}+00$ & $8.20 \mathrm{E}-13$ & $1.00 \mathrm{E}-09$ & $1.60 \mathrm{E}-09$ \\
\hline & PR-144D & CE-144 & $4.20 \mathrm{E}-09$ & $3.70 \mathrm{E}-10$ & $0.00 \mathrm{E}+00$ & $0.00 \mathrm{E}+00$ & $6.20 \mathrm{E}-10$ & $8.80 \mathrm{E}-07$ & $8.85 \mathrm{E}-07$ \\
\hline & Total & & $9.42 \mathrm{E}-05$ & $1.21 \mathrm{E}-02$ & $4.21 \mathrm{E}-04$ & 1.99E-07 & $2.19 \mathrm{E}-04$ & $3.80 \mathrm{E}-06$ & $1.28 \mathrm{E}-02$ \\
\hline
\end{tabular}

*Cld. Gamma=gamma cloud shine, Dep. Gamma=gamma deposition, Resus. = re-suspension, Cld. Beta=Beta cloud shine, Dep. Beta=Beta deposition

Table 6. Results of unshielded external dose rates calculation in year 1 with PC-CREAM in 5 distances around the TRR, $(\mu \mathrm{Sv} / \mathrm{y})$

\begin{tabular}{|c|c|c|c|c|c|c|c|c|}
\hline & Nuclide & Parent & Cld. Gamma & Dep. Gamma & Resus. & Cld beta & Dep beta & Total \\
\hline \multirow{13}{*}{ 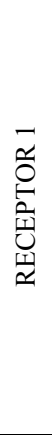 } & AR-41 & & $8.90 \mathrm{E}-01$ & $0.00 \mathrm{E}+00$ & $0.00 \mathrm{E}+00$ & $3.00 \mathrm{E}-03$ & $0.00 \mathrm{E}+00$ & $8.93 \mathrm{E}-01$ \\
\hline & CO-60 & & $4.00 \mathrm{E}-05$ & $2.10 \mathrm{E}-02$ & $1.80 \mathrm{E}-02$ & $1.30 \mathrm{E}-08$ & $0.00 \mathrm{E}+00$ & $3.90 \mathrm{E}-02$ \\
\hline & KR-87 & & $2.30 \mathrm{E}-02$ & $0.00 \mathrm{E}+00$ & $0.00 \mathrm{E}+00$ & $3.60 \mathrm{E}-04$ & $0.00 \mathrm{E}+00$ & $2.34 \mathrm{E}-02$ \\
\hline & KR-88 & & $5.60 \mathrm{E}-02$ & $0.00 \mathrm{E}+00$ & $0.00 \mathrm{E}+00$ & $1.00 \mathrm{E}-04$ & $0.00 \mathrm{E}+00$ & $5.61 \mathrm{E}-02$ \\
\hline & SR-90 & & $0.00 \mathrm{E}+00$ & $1.40 \mathrm{E}-11$ & $5.10 \mathrm{E}-04$ & $8.00 \mathrm{E}-10$ & $4.10 \mathrm{E}-06$ & 5.14E-04 \\
\hline & I-131 & & $7.40 \mathrm{E}-08$ & $9.40 \mathrm{E}-06$ & $2.50 \mathrm{E}-04$ & $3.50 \mathrm{E}-10$ & $5.50 \mathrm{E}-06$ & $2.65 \mathrm{E}-04$ \\
\hline & I-133 & & $4.60 \mathrm{E}-07$ & $7.00 \mathrm{E}-06$ & $1.60 \mathrm{E}-04$ & $3.00 \mathrm{E}-09$ & $1.50 \mathrm{E}-04$ & $3.17 \mathrm{E}-04$ \\
\hline & CS-134 & & $7.60 \mathrm{E}-06$ & $3.60 \mathrm{E}-03$ & $4.90 \mathrm{E}-03$ & 7.70E-09 & $4.50 \mathrm{E}-05$ & $8.55 \mathrm{E}-03$ \\
\hline & $\begin{array}{l}\text { CS-137 } \\
\end{array}$ & & $6.90 \mathrm{E}-06$ & $3.80 \mathrm{E}-03$ & $1.30 \mathrm{E}-02$ & $2.80 \mathrm{E}-08$ & $2.00 \mathrm{E}-04$ & $1.70 \mathrm{E}-02$ \\
\hline & CE-144 & & $4.10 \mathrm{E}-08$ & $3.70 \mathrm{E}-05$ & $1.80 \mathrm{E}-03$ & $1.30 \mathrm{E}-09$ & $0.00 \mathrm{E}+00$ & $1.84 \mathrm{E}-03$ \\
\hline & Y-90D & SR-90 & $7.70 \mathrm{E}-18$ & $0.00 \mathrm{E}+00$ & $0.00 \mathrm{E}+00$ & $2.10 \mathrm{E}-12$ & $1.80 \mathrm{E}-08$ & $1.80 \mathrm{E}-08$ \\
\hline & PR-144D & CE-144 & $4.50 \mathrm{E}-09$ & $0.00 \mathrm{E}+00$ & $0.00 \mathrm{E}+00$ & $2.20 \mathrm{E}-09$ & $1.90 \mathrm{E}-05$ & $1.90 \mathrm{E}-05$ \\
\hline & Total & & $9.69 \mathrm{E}-01$ & $2.85 \mathrm{E}-02$ & $3.86 \mathrm{E}-02$ & $3.46 \mathrm{E}-03$ & $4.24 \mathrm{E}-04$ & $1.04 E+00$ \\
\hline \multirow{13}{*}{ 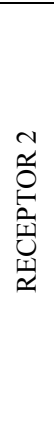 } & AR-41 & & $5.20 \mathrm{E}-01$ & $0.00 \mathrm{E}+00$ & $0.00 \mathrm{E}+00$ & $2.10 \mathrm{E}-03$ & $0.00 \mathrm{E}+00$ & $5.22 \mathrm{E}-01$ \\
\hline & CO-60 & & $2.40 \mathrm{E}-05$ & $1.30 \mathrm{E}-02$ & $1.10 \mathrm{E}-02$ & $8.70 \mathrm{E}-09$ & $0.00 \mathrm{E}+00$ & $2.40 \mathrm{E}-02$ \\
\hline & KR-87 & & $1.40 \mathrm{E}-02$ & $0.00 \mathrm{E}+00$ & $0.00 \mathrm{E}+00$ & $2.50 \mathrm{E}-04$ & $0.00 \mathrm{E}+00$ & $1.43 \mathrm{E}-02$ \\
\hline & KR-88 & & $3.30 \mathrm{E}-02$ & $0.00 \mathrm{E}+00$ & $0.00 \mathrm{E}+00$ & $7.00 \mathrm{E}-05$ & $0.00 \mathrm{E}+00$ & $3.31 \mathrm{E}-02$ \\
\hline & SR-90 & & $0.00 \mathrm{E}+00$ & $8.60 \mathrm{E}-12$ & $3.20 \mathrm{E}-04$ & $5.50 \mathrm{E}-10$ & $2.60 \mathrm{E}-06$ & $3.23 \mathrm{E}-04$ \\
\hline & I-131 & & $4.20 \mathrm{E}-08$ & $6.20 \mathrm{E}-06$ & $1.70 \mathrm{E}-04$ & $2.40 \mathrm{E}-10$ & $3.70 \mathrm{E}-06$ & $1.80 \mathrm{E}-04$ \\
\hline & I-133 & & $2.60 \mathrm{E}-07$ & $4.70 \mathrm{E}-06$ & $1.10 \mathrm{E}-04$ & $2.00 \mathrm{E}-09$ & $1.00 \mathrm{E}-04$ & $2.15 \mathrm{E}-04$ \\
\hline & CS-134 & & $4.40 \mathrm{E}-06$ & $2.30 \mathrm{E}-03$ & $3.00 \mathrm{E}-03$ & $5.20 \mathrm{E}-09$ & $2.80 \mathrm{E}-05$ & $5.33 \mathrm{E}-03$ \\
\hline & $\begin{array}{l}\text { CS-137 } \\
\end{array}$ & & $4.00 \mathrm{E}-06$ & $2.40 \mathrm{E}-03$ & $8.00 \mathrm{E}-03$ & $1.90 \mathrm{E}-08$ & $1.20 \mathrm{E}-04$ & $1.05 \mathrm{E}-02$ \\
\hline & CE-144 & & $2.30 \mathrm{E}-08$ & $2.30 \mathrm{E}-05$ & $1.10 \mathrm{E}-03$ & $8.70 \mathrm{E}-10$ & $0.00 \mathrm{E}+00$ & $1.12 \mathrm{E}-03$ \\
\hline & Y-90D & SR-90 & $7.50 \mathrm{E}-18$ & $0.00 \mathrm{E}+00$ & $0.00 \mathrm{E}+00$ & $2.00 \mathrm{E}-12$ & $1.70 \mathrm{E}-08$ & $1.70 \mathrm{E}-08$ \\
\hline & PR-144D & CE-144 & $4.20 \mathrm{E}-09$ & $0.00 \mathrm{E}+00$ & $0.00 \mathrm{E}+00$ & $2.10 \mathrm{E}-09$ & $1.80 \mathrm{E}-05$ & $1.80 \mathrm{E}-05$ \\
\hline & Total & & $5.67 \mathrm{E}-01$ & $1.77 \mathrm{E}-02$ & $2.37 \mathrm{E}-02$ & $2.42 \mathrm{E}-03$ & $2.72 \mathrm{E}-04$ & $6.11 \mathrm{E}-01$ \\
\hline
\end{tabular}




\begin{tabular}{|c|c|c|c|c|c|c|c|c|}
\hline \multirow{13}{*}{ 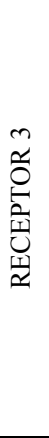 } & AR-41 & & $1.30 \mathrm{E}-01$ & $0.00 \mathrm{E}+00$ & $0.00 \mathrm{E}+00$ & $5.70 \mathrm{E}-04$ & $0.00 \mathrm{E}+00$ & $1.31 \mathrm{E}-01$ \\
\hline & CO-60 & & $6.20 \mathrm{E}-06$ & $4.10 \mathrm{E}-03$ & $3.40 \mathrm{E}-03$ & $2.40 \mathrm{E}-09$ & $0.00 \mathrm{E}+00$ & $7.51 \mathrm{E}-03$ \\
\hline & KR-87 & & $3.40 \mathrm{E}-03$ & $0.00 \mathrm{E}+00$ & $0.00 \mathrm{E}+00$ & $6.70 \mathrm{E}-05$ & $0.00 \mathrm{E}+00$ & $3.47 \mathrm{E}-03$ \\
\hline & KR-88 & & $8.60 \mathrm{E}-03$ & $0.00 \mathrm{E}+00$ & $0.00 \mathrm{E}+00$ & $1.90 \mathrm{E}-05$ & $0.00 \mathrm{E}+00$ & $8.62 \mathrm{E}-03$ \\
\hline & SR-90 & & $0.00 \mathrm{E}+00$ & $2.70 \mathrm{E}-12$ & $1.00 \mathrm{E}-04$ & $1.50 \mathrm{E}-10$ & $8.10 \mathrm{E}-07$ & $1.01 \mathrm{E}-04$ \\
\hline & I-131 & & $1.00 \mathrm{E}-08$ & $1.80 \mathrm{E}-06$ & 4.80E-05 & $6.60 \mathrm{E}-11$ & $1.10 \mathrm{E}-06$ & $5.09 \mathrm{E}-05$ \\
\hline & I-133 & & $6.60 \mathrm{E}-08$ & $1.30 \mathrm{E}-06$ & $3.10 \mathrm{E}-05$ & $5.60 \mathrm{E}-10$ & $2.90 \mathrm{E}-05$ & $6.14 \mathrm{E}-05$ \\
\hline & CS-134 & & $1.10 \mathrm{E}-06$ & $7.00 \mathrm{E}-04$ & $9.50 \mathrm{E}-04$ & $1.50 \mathrm{E}-09$ & $8.80 \mathrm{E}-06$ & $1.66 \mathrm{E}-03$ \\
\hline & CS-137 & & $1.00 \mathrm{E}-06$ & $7.50 \mathrm{E}-04$ & $2.50 \mathrm{E}-03$ & $5.30 \mathrm{E}-09$ & $3.80 \mathrm{E}-05$ & $3.29 \mathrm{E}-03$ \\
\hline & CE-144 & & $5.70 \mathrm{E}-09$ & $7.20 \mathrm{E}-06$ & $3.50 \mathrm{E}-04$ & $2.40 \mathrm{E}-10$ & $0.00 \mathrm{E}+00$ & $3.57 \mathrm{E}-04$ \\
\hline & Y-90D & SR-90 & $4.30 \mathrm{E}-18$ & $0.00 \mathrm{E}+00$ & $0.00 \mathrm{E}+00$ & $1.10 \mathrm{E}-12$ & $1.20 \mathrm{E}-08$ & $1.20 \mathrm{E}-08$ \\
\hline & PR-144D & CE-144 & $2.40 \mathrm{E}-09$ & $0.00 \mathrm{E}+00$ & $0.00 \mathrm{E}+00$ & $1.00 \mathrm{E}-09$ & $1.20 \mathrm{E}-05$ & $1.20 \mathrm{E}-05$ \\
\hline & Total & & $1.42 \mathrm{E}-01$ & $5.56 \mathrm{E}-03$ & $7.38 \mathrm{E}-03$ & $6.56 \mathrm{E}-04$ & $8.97 \mathrm{E}-05$ & $1.56 \mathrm{E}-01$ \\
\hline \multirow{13}{*}{ 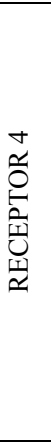 } & AR-41 & & $6.50 \mathrm{E}-02$ & $0.00 \mathrm{E}+00$ & $0.00 \mathrm{E}+00$ & $3.00 \mathrm{E}-04$ & $0.00 \mathrm{E}+00$ & $1.20 \mathrm{E}-05$ \\
\hline & CO-60 & & $3.20 \mathrm{E}-06$ & $2.40 \mathrm{E}-03$ & $2.00 \mathrm{E}-03$ & $1.30 \mathrm{E}-09$ & $0.00 \mathrm{E}+00$ & $6.53 \mathrm{E}-02$ \\
\hline & KR-87 & & $1.70 \mathrm{E}-03$ & $0.00 \mathrm{E}+00$ & $0.00 \mathrm{E}+00$ & $3.50 \mathrm{E}-05$ & $0.00 \mathrm{E}+00$ & $4.40 \mathrm{E}-03$ \\
\hline & KR-88 & & $4.30 \mathrm{E}-03$ & $0.00 \mathrm{E}+00$ & $0.00 \mathrm{E}+00$ & $1.00 \mathrm{E}-05$ & $0.00 \mathrm{E}+00$ & $1.74 \mathrm{E}-03$ \\
\hline & SR-90 & & $0.00 \mathrm{E}+00$ & $1.50 \mathrm{E}-12$ & $5.70 \mathrm{E}-05$ & $8.40 \mathrm{E}-11$ & $4.70 \mathrm{E}-07$ & $4.31 \mathrm{E}-03$ \\
\hline & $\mathrm{I}-131$ & & $5.20 \mathrm{E}-09$ & $9.70 \mathrm{E}-07$ & $2.60 \mathrm{E}-05$ & $3.50 \mathrm{E}-11$ & $5.70 \mathrm{E}-07$ & $5.75 \mathrm{E}-05$ \\
\hline & I-133 & & $3.30 \mathrm{E}-08$ & $7.20 \mathrm{E}-07$ & $1.70 \mathrm{E}-05$ & $3.00 \mathrm{E}-10$ & $1.60 \mathrm{E}-05$ & $2.75 \mathrm{E}-05$ \\
\hline & CS-134 & & $5.70 \mathrm{E}-07$ & $4.10 \mathrm{E}-04$ & $5.50 \mathrm{E}-04$ & $8.00 \mathrm{E}-10$ & $5.00 \mathrm{E}-06$ & $3.38 \mathrm{E}-05$ \\
\hline & CS-137 & & $5.10 \mathrm{E}-07$ & $4.30 \mathrm{E}-04$ & $1.40 \mathrm{E}-03$ & $2.90 \mathrm{E}-09$ & $2.20 \mathrm{E}-05$ & $9.66 \mathrm{E}-04$ \\
\hline & CE-144 & & $2.90 \mathrm{E}-09$ & $4.10 \mathrm{E}-06$ & $2.00 \mathrm{E}-04$ & $1.30 \mathrm{E}-10$ & $0.00 \mathrm{E}+00$ & $1.85 \mathrm{E}-03$ \\
\hline & Y-90D & SR-90 & $3.40 \mathrm{E}-18$ & $0.00 \mathrm{E}+00$ & $0.00 \mathrm{E}+00$ & $9.20 \mathrm{E}-13$ & $1.10 \mathrm{E}-08$ & $2.04 \mathrm{E}-04$ \\
\hline & PR-144D & CE-144 & $1.70 \mathrm{E}-09$ & $0.00 \mathrm{E}+00$ & $0.00 \mathrm{E}+00$ & $7.50 \mathrm{E}-10$ & $9.90 \mathrm{E}-06$ & $1.10 \mathrm{E}-08$ \\
\hline & Total & & $7.10 \mathrm{E}-02$ & $3.25 \mathrm{E}-03$ & $4.25 \mathrm{E}-03$ & $3.45 \mathrm{E}-04$ & $6.60 \mathrm{E}-05$ & $9.90 \mathrm{E}-06$ \\
\hline \multirow{13}{*}{ 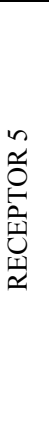 } & AR-41 & & $4.00 \mathrm{E}-02$ & $0.00 \mathrm{E}+00$ & $0.00 \mathrm{E}+00$ & $1.90 \mathrm{E}-04$ & $0.00 \mathrm{E}+00$ & $4.02 \mathrm{E}-02$ \\
\hline & CO-60 & & $2.00 \mathrm{E}-06$ & $1.60 \mathrm{E}-03$ & $1.40 \mathrm{E}-03$ & $8.70 \mathrm{E}-10$ & $0.00 \mathrm{E}+00$ & $3.00 \mathrm{E}-03$ \\
\hline & KR-87 & & $1.00 \mathrm{E}-03$ & $0.00 \mathrm{E}+00$ & $0.00 \mathrm{E}+00$ & $2.20 \mathrm{E}-05$ & $0.00 \mathrm{E}+00$ & $1.02 \mathrm{E}-03$ \\
\hline & KR-88 & & $2.70 \mathrm{E}-03$ & $0.00 \mathrm{E}+00$ & $0.00 \mathrm{E}+00$ & $6.70 \mathrm{E}-06$ & $0.00 \mathrm{E}+00$ & $2.71 \mathrm{E}-03$ \\
\hline & SR-90 & & $0.00 \mathrm{E}+00$ & $1.10 \mathrm{E}-12$ & $4.00 \mathrm{E}-05$ & $5.50 \mathrm{E}-11$ & $3.20 \mathrm{E}-07$ & $4.03 \mathrm{E}-05$ \\
\hline & I-131 & & $3.30 \mathrm{E}-09$ & $6.40 \mathrm{E}-07$ & $1.70 \mathrm{E}-05$ & $2.30 \mathrm{E}-11$ & $3.80 \mathrm{E}-07$ & $1.80 \mathrm{E}-05$ \\
\hline & I-133 & & $2.10 \mathrm{E}-08$ & $4.80 \mathrm{E}-07$ & $1.10 \mathrm{E}-05$ & $1.90 \mathrm{E}-10$ & $1.00 \mathrm{E}-05$ & $2.15 \mathrm{E}-05$ \\
\hline & CS-134 & & $3.60 \mathrm{E}-07$ & $2.80 \mathrm{E}-04$ & $3.80 \mathrm{E}-04$ & $5.20 \mathrm{E}-10$ & $3.50 \mathrm{E}-06$ & 6.64E-04 \\
\hline & CS-137 & & $3.30 \mathrm{E}-07$ & $3.00 \mathrm{E}-04$ & $9.90 \mathrm{E}-04$ & $1.90 \mathrm{E}-09$ & $1.50 \mathrm{E}-05$ & $1.31 \mathrm{E}-03$ \\
\hline & CE-144 & & $1.80 \mathrm{E}-09$ & $2.90 \mathrm{E}-06$ & $1.40 \mathrm{E}-04$ & $8.70 \mathrm{E}-11$ & $0.00 \mathrm{E}+00$ & $1.43 \mathrm{E}-04$ \\
\hline & Y-90D & SR-90 & $3.00 \mathrm{E}-18$ & $0.00 \mathrm{E}+00$ & $0.00 \mathrm{E}+00$ & $8.20 \mathrm{E}-13$ & $1.00 \mathrm{E}-08$ & $1.00 \mathrm{E}-08$ \\
\hline & PR-144D & CE-144 & $1.30 \mathrm{E}-09$ & $0.00 \mathrm{E}+00$ & $0.00 \mathrm{E}+00$ & $6.20 \mathrm{E}-10$ & $8.80 \mathrm{E}-06$ & $8.80 \mathrm{E}-06$ \\
\hline & Total & & 4.37E-02 & $2.18 E-03$ & $2.98 \mathrm{E}-03$ & $2.19 \mathrm{E}-04$ & $3.80 \mathrm{E}-05$ & 4.91E-02 \\
\hline
\end{tabular}

The portion of different exposure pathways in the total committed dose are given in Fig. 2. According to the results, the most important pathway for the dose received is cloud gamma having value of 95.9 percent. For the other nuclides not included because they contribute less than 1 percent.

Different dose percentage of radionuclide in $300 \mathrm{~m}$ away from the reactor stack and dominant direction for adult are presented in the Fig. 3. It shows that the most effective radionuclides for internal dose exposure in decreasing order are Ar-41, Kr-88, $\mathrm{Kr}-87$ and $\mathrm{Co}-60$. For the other nuclides not included because they contribute less than 1 percent.

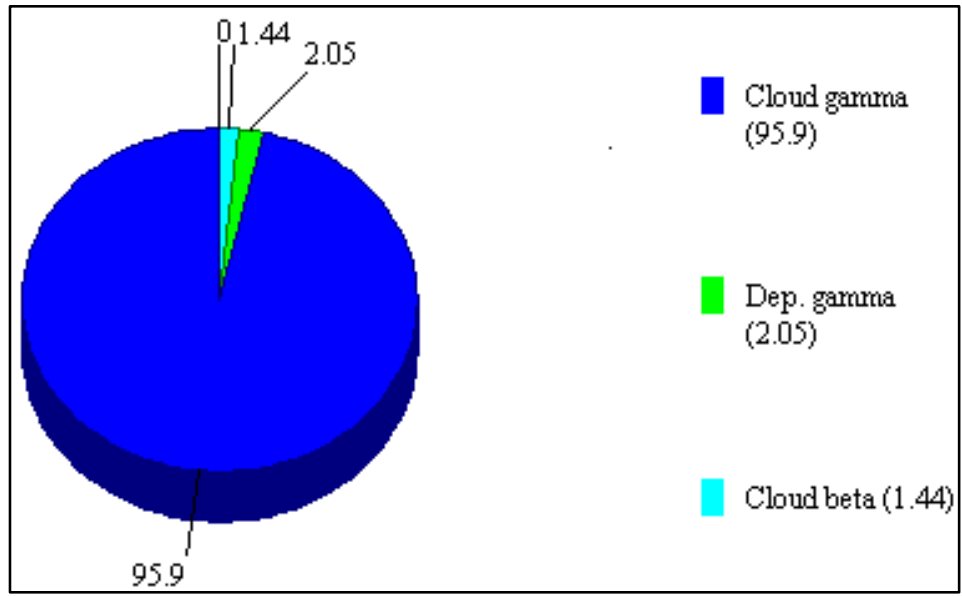

Fig. 2. Percentage of different exposure pathways in the total committed dose 


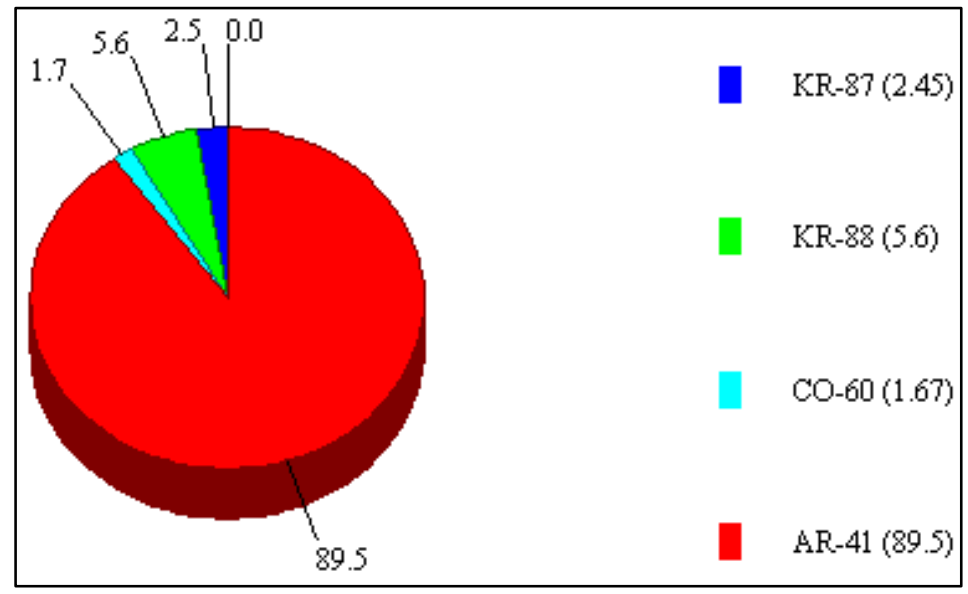

Fig. 3. Different dose percentage of radionuclide in $300 \mathrm{~m}$ away from the reactor stack and dominant direction for adult.

The results of area monitoring in the vicinity of TRR which achieved by TLD detector are shown in Table 7. The average annual effective dose equivalents from the TLD analyzing were found to be from $113 \mu \mathrm{Sv}$ up to $150 \mu \mathrm{Sv}$ in TRR site from all facilities. It is in good conformity with measured outdoor gamma radiation data from the online monitoring in Tehran province were found to be about $125 \mu \mathrm{Sv}$ [11]. We must notice that there are some facilities which are using radioactive material for laboratories or research activities and all of them are placed in TRR site. So results of TLD detectors are based on from all of these facilities probable releases.

Table 7. Results of TLD analyzing

\begin{tabular}{cccc}
\hline Groups & ID & Reading $(\mathrm{nC})$ & Dose $(\mu \mathrm{Sv})$ \\
\hline \multirow{3}{*}{ A } & A1 & 65.61 & 126.594 \\
& A2 & 67.97 & 133.986 \\
& A3 & 60.65 & 119.729 \\
\hline \multirow{3}{*}{ B } & B1 & 67.07 & 128.660 \\
& B2 & 73.11 & 137.832 \\
& B3 & 68.64 & 127.952 \\
\hline \multirow{3}{*}{ C } & C1 & 71.43 & 138.502 \\
& C2 & 77.40 & 150.077 \\
& C3 & 78.19 & 143.194 \\
\hline \multirow{2}{*}{ D } & D1 & 55.15 & 113.007 \\
& D2 & 69.14 & 130.623 \\
& D3 & 61.97 & 119.453 \\
\hline \multirow{2}{*}{ E } & E1 & 66.67 & 128.450 \\
& E2 & 79.39 & 147.109 \\
\hline \multirow{2}{*}{ F } & E3 & 63.98 & 125.999 \\
\hline & F1 & 69.67 & 129.449 \\
& F2 & 67.76 & 127.308 \\
\hline
\end{tabular}

The results of soil and leaves sampling are presented in table 8 and 9 respectively. Sampling analysis of soil and leaves from TRR site areas shows just natural radionuclides in the background level according to TRR site area. 
Table 8. Results of soil sampling and laboratory analyzing

\begin{tabular}{|c|c|c|c|c|c|c|c|}
\hline \multirow{2}{*}{ Sample station } & \multicolumn{4}{|c|}{ Sample Activity $(\mathrm{Bq} / \mathrm{Kg})$} & \multirow{2}{*}{$\begin{array}{c}\text { Sample volume } \\
\text { (gr) }\end{array}$} & \multirow{2}{*}{$\begin{array}{c}\text { Background } \\
\text { Activity }(\mathrm{Bq} / \mathrm{Kg})^{\{6,7\}}\end{array}$} & \\
\hline & Ra-226 & Cs-137 & $\mathrm{K}-40$ & Th-232 & & & \\
\hline $\mathrm{NE}$ & $30 \pm 4$ & $2.6 \pm 0.1$ & $771 \pm 82$ & $32 \pm 2$ & 500 & \multirow{8}{*}{$.29-28.82$} & \\
\hline $\mathrm{N}$ & $31 \pm 4$ & $1.3 \pm 0.08$ & $900 \pm 103$ & $38 \pm 5$ & 500 & & 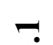 \\
\hline NW & $26 \pm 3$ & $0.5 \pm 0.04$ & $604 \pm 75$ & $27 \pm 3$ & 500 & & 0 \\
\hline $\mathrm{W}$ & $21 \pm 2$ & $0.8 \pm 0.07$ & $601 \pm 73$ & $25 \pm 2.5$ & 500 & & $\widehat{\theta}$ \\
\hline SW & $25 \pm 2$ & $<$ MDA & $761 \pm 68$ & $29 \pm 2.5$ & 500 & & $\sum$ \\
\hline $\mathrm{S}$ & $22.5 \pm 2$ & $1.5 \pm 0.1$ & $553 \pm 52$ & $29 \pm 3$ & 500 & & \\
\hline SE & $24 \pm 2.5$ & $1 \pm 0.1$ & $595 \pm 56$ & $25 \pm 2$ & 500 & & \\
\hline E & $22 \pm 2$ & $0.6 \pm 0.05$ & $625 \pm 60$ & $27 \pm 3$ & 500 & & \\
\hline
\end{tabular}

$*$ MDA (Minimum Detectable Activity)

Table 9. Results of tree's leaf sampling and laboratory analyzing

\begin{tabular}{|c|c|c|c|}
\hline Station & Sample Type & Elements & Activity $(\mathrm{Bq} / \mathrm{Kg})$ \\
\hline \multirow{8}{*}{ NE } & \multirow{2}{*}{ Wet Leaves } & $\mathrm{Be} 7$ & $1.51 \mathrm{E}+2$ \\
\hline & & K40 & $3.14 \mathrm{E}+2$ \\
\hline & \multirow{2}{*}{ Dry Leaves } & $\mathrm{Be} 7$ & $7.0 \mathrm{E}+1$ \\
\hline & & K40 & $1.8 \mathrm{E}+2$ \\
\hline & \multirow{2}{*}{ Wet Leaves } & $\mathrm{Be} 7$ & $2.9 \mathrm{E}+1$ \\
\hline & & K40 & $8.3 \mathrm{E}+1$ \\
\hline & \multirow{2}{*}{ Dry Leaves } & $\mathrm{Be} 7$ & $3.9 \mathrm{E}+1$ \\
\hline & & K40 & $1.5 \mathrm{E}+2$ \\
\hline \multirow{8}{*}{$\mathrm{N}$} & \multirow{2}{*}{ Wet Leaves } & $\mathrm{Be} 7$ & $1.1 \mathrm{E}+2$ \\
\hline & & K40 & $2.4 \mathrm{E}+2$ \\
\hline & \multirow{2}{*}{ Dry Leaves } & $\mathrm{Be} 7$ & $6.0 \mathrm{E}+1$ \\
\hline & & K40 & $1.9 \mathrm{E}+2$ \\
\hline & \multirow{2}{*}{ Wet Leaves } & $\mathrm{Be} 7$ & $1.9 \mathrm{E}+1$ \\
\hline & & K40 & $9.2 \mathrm{E}+1$ \\
\hline & \multirow{2}{*}{ Dry Leaves } & $\mathrm{Be} 7$ & $3.6 \mathrm{E}+1$ \\
\hline & & K40 & $1.9 \mathrm{E}+2$ \\
\hline \multirow{8}{*}{ NW } & \multirow{2}{*}{ Wet Leaves } & $\star^{2}$ & $\star$ \\
\hline & & * & $\star$ \\
\hline & \multirow{2}{*}{ Dry Leaves } & $\mathrm{Be} 7$ & $2.4 \mathrm{E}+1$ \\
\hline & & K40 & $2.2 \mathrm{E}+2$ \\
\hline & \multirow{2}{*}{ Wet Leaves } & $\mathrm{Be} 7$ & $2.3 \mathrm{E}+1$ \\
\hline & & K40 & $1.1 \mathrm{E}+2$ \\
\hline & \multirow{2}{*}{ Dry Leaves } & $\mathrm{Be} 7$ & $3.1 \mathrm{E}+1$ \\
\hline & & K40 & $1.9 \mathrm{E}+2$ \\
\hline \multirow{8}{*}{ SW } & \multirow{2}{*}{ Wet Leaves } & * & * \\
\hline & & * & * \\
\hline & \multirow{2}{*}{ Dry Leaves } & $\mathrm{Be} 7$ & $6.8 \mathrm{E}+1$ \\
\hline & & K40 & $1.5 \mathrm{E}+2$ \\
\hline & \multirow{2}{*}{ Wet Leaves } & $\mathrm{Be} 7$ & $2.5 \mathrm{E}+1$ \\
\hline & & K40 & $1.0 \mathrm{E}+2$ \\
\hline & \multirow{2}{*}{ Dry Leaves } & $\mathrm{Be} 7$ & $4.0 \mathrm{E}+1$ \\
\hline & & K40 & $2.5 \mathrm{E}+2$ \\
\hline \multirow{8}{*}{$\mathrm{S}$} & \multirow{2}{*}{ Wet Leaves } & $*$ & $*$ \\
\hline & & * & * \\
\hline & \multirow{2}{*}{ Dry Leaves } & $*$ & $*$ \\
\hline & & $*$ & $*$ \\
\hline & Wet I eaves & $\mathrm{Be} 7$ & $2.1 \mathrm{E}+1$ \\
\hline & Wet Leaves & K40 & $1.2 \mathrm{E}+2$ \\
\hline & Dry Leaves & $\mathrm{Be} 7$ & $3.5 \mathrm{E}+1$ \\
\hline & Dry Leaves & K40 & $1.9 \mathrm{E}+2$ \\
\hline & & * & * \\
\hline & Wet Leaves & * & * \\
\hline & Dry Leaves & $\mathrm{Be} 7$ & $6.6 \mathrm{E}+1$ \\
\hline SF & Diy Leaves & K40 & $2.3 \mathrm{E}+2$ \\
\hline SE & & $\mathrm{Be} 7$ & $3.0 \mathrm{E}+1$ \\
\hline & Wet Leaves & K40 & $8.3 \mathrm{E}+1$ \\
\hline & Drv Leaves & $\mathrm{Be} 7$ & $4.1 \mathrm{E}+1$ \\
\hline & Dry Leaves & K40 & $1.5 \mathrm{E}+2$ \\
\hline
\end{tabular}




\section{Conclusions}

A comparison of activity concentrations in air which calculated by PC-CREAM 98 with the Annual Limits on Derived Air Concentrations (DACs) in NRC Regulations (10 CFR part 20) show that, the effluent concentration in air from the TRR discharge are negligible and even below the limited levels [12]. Also the result of the individual dose indicate that, higher dose for all receptors is smaller than the dose constraint of $0.1 \mathrm{mSv} / \mathrm{y}$ which determined by the Iranian Nuclear Regulatory Authority (INRA).

The average annual effective doses equivalents from the TLD analyzing show that, the outdoor gamma radiation around TRR is at the background level. Also there is no any considerable radionuclide in soil and tree's leaf samples (artificial or natural) and analyzing results show just natural radioactivity at the background level.

According to the computer modeling and experimental measurements results, it can be concluded that the effective doses of public around the TRR during normal operation are smaller than the dose constraint limit $(0.1 \mathrm{mSv} / \mathrm{y})$. Therefore, the required safety with regard to radiation in Tehran Research Reactor is guaranteed. Consequently, the reactor operates in good situation and safe manner.

\section{References}

[1] Mehdi Sohrabi, Zeinab Parsouzi A.Sh, Reza Amrollahi, Maryam Ghasemi, Public exposure from environmental release of radioactive material under normal operation of unit-1 Bushehr nuclear power plant, Annals Of Nuclear Energy 55(351-358), May 2013.

[2] Alastair G. Williams, Geoffrey H. Clark and Leisa Dyer, Nuclear tools for characterizing radiological dispersion in complex terrain: evaluation of regulatory and emergency response models, Int. J. Environment and Pollution, Vol. 24, Nos. 1/2/3/4, 2005.

[3] A.M.Esposito, C. Sabbarese, C. Sirignano, L. Visciano, A. D’Onofrio, C. Lubritto, F. Terrasi, S. Alfieri, G. Migliore, Preliminary Study Of The Environmental Radiological Assessment For The Garigliano Nuclear Power Plant Decommissioning, information on: http://www.iaea.org/inis/collection/NCLCollectionStore/_Public/37/115/37115723.pdf

[4] Assessment of radiological impact of routine and accidental atmospheric releases of radionuclides to the living environment, Institute of Information Theory and Automation, Academy of Sciences of the Czech Republic, Prague, information on: http://havarrp.utia.cas.cz/eng/reporty_PDF/Varna_EGP.pdf

[5] Smith, J.G., Simmonds, J.R., The methodology for assessing the radiological consequences of routine release of radionuclides to the environment used in Pc-Cream 08. Health Protection Agency (HPA), 2009.

[6] Simmonds, J.R., Lawson, G., Mayall, A., methodology for assessing the radiological consequences of routine releases of radionuclides to the environment, EC, Luxembourg, International Committee on Radiation protection 72, EUR 15760, 1995.

[7] AEOI, Atomic Energy Organization of Iran, Safety Analysis Report for Tehran Research Reactor (TRR), 2009.

[8] AEOI, Atomic Energy Organization of Iran, Environmental Assessment for Tehran Research Reactor (TRR), 2008.

[9] UNSCEAR, United Nation Scientific Committee on the Effects of Atomic Radiation Sources and effects of ionizing radiation, Report of the United Nations Scientific Committee on the Effect of Atomic Radiation to General Assembly, New York, USA, 2000.

[10] IAEA, International Atomic Energy Agency, Measurement of Radionuclides in Food and the Environment, Technical Report Series No.295, 1989.

[11] Hafezi, S., Amidi, J., Attarilar, A., Concentration of natural radionuclides in Soil and assessment of external exposure to the public in Tehran. J. Radiat. Res 3(2): 85-88, 2005.

[12] NRC, Nuclear Regulatory Commission, Annual Limits on Intake (ALIs) and Derived Air Concentrations (DACs) of Radionuclides for Occupational Exposure; Effluent Concentrations; Concentrations for Release to Sewerage. 10 CFR Part 20, Appendix B, 1996. 